\title{
ORIGINAL ARTICLE \\ Diagnostic transcranial magnetic stimulation as a prognostic tool in children with acute transverse myelitis
}

\author{
VB Voitenkov ${ }^{1}$, AV Klimkin ${ }^{1}$, NV Skripchenko², NF Pulman ${ }^{2}$ and MV Ivanova ${ }^{2}$
}

Study design: We performed transcranial magnetic stimulation (TMS) in children with sequelae of acute transverse myelitis. Single-pulse TMS protocol was implemented. Twenty controls and 24 patients with myelitis were enrolled. TMS was performed on 12-24 day after the onset of the first symptoms, average on 16th day.

Objectives: The objective of our study was to evaluate motor pathways in children with viral myelitis using the TMS technique.

Setting: All investigations were performed in Scientific Research Institute for Children's Infections, Russia.

Results: There were statistically significant differences between the groups on central motor conduction time and motor evoked potential (MEPs) amplitudes. MEP thresholds were elevated, and MEP shape was abnormal in 96\% of patients with myelitis ( $n=23$ ). Three neurophysiologic patterns were observed: the presence of both cortical and spinal MEP, the absence of cortical MEP with the spinal MEP present and the total absence of both cortical and spinal MEP. Last finding was associated with paraplegia, resistant to any sort of treatment.

Conclusion: Thus, myelitis in $96 \%$ of the cases causes neurophysiologic changes, which may be detected by TMS; the method may be used as a predicting tool.

Spinal Cord (2016) 54, 226-228; doi:10.1038/sc.2015.129; published online 4 August 2015

\section{INTRODUCTION}

Acute transverse myelitis is the infrequent, severe and potentially devastating condition that has been estimated to occur with an annual worldwide incidence of 4 cases per 100000 individuals. ${ }^{1}$ In pediatric population, it often results in motor, sensory and autonomic dysfunction, with growing demand for reliable diagnostic and prognostic tools. ${ }^{2}$

Clinical examination in children is sometimes unreliable, and standard neurophysiologic tests could be insufficient in setting precise diagnosis; thus, transcranial magnetic stimulation (TMS) is considered as one of the valuable diagnostic methods. ${ }^{3,4}$ TMS is a method for focal noninvasive brain stimulation that is based on the principles of electromagnetic induction. ${ }^{5}$ TMS is an effective diagnostic and a therapeutic technique with applications in neurology, psychiatry and rehabilitation medicine, which can provide insight into developmental neurology and neurophysiology in children. ${ }^{6}$ TMS can detect corticospinal tract abnormalities not identified by neuroimaging., ${ }^{3,4} 6$

In the patients with compressive myelopathies, TMS was found to be helpful in determining the functional significance of neuroimaging findings. TMS can supplement clinical examination and neuroimaging findings also in the assessment of the spinal cord injury level., However, reports of TMS use in pediatric acute myelitis are relatively rare, and mostly such works are case reports or small series consisting of 2-3 patients. ${ }^{7}$ We have performed the investigation of corticospinal pathway characteristics in children with acute myelitis, with special attention toward clinical significance of TMS and its prognostic value in this case.
The objective of our study was to evaluate motor pathways in children with viral myelitis using the TMS technique.

\section{MATERIALS AND METHODS}

Twenty healthy children (7-14 years old, average 12 years, 7 females, 13 males) without any signs of spinal disorders were enrolled as controls. Main group consisted of 24 patients ( $8-16$ years old, average 11 years, 14 females, 10 males) with acute viral transverse myelitis. In 4 cases, cervical localization of the lesion -in 7 thoracic and in 13 lumbar-was diagnosed. Etiology in all cases was viral, in 20 cases enterovirus and in 4 cases Epstein-Barr virus.

Eighteen children were paraplegic, and 6 cases were paraparetic; among 7 patients with cervical myelitis, 2 were tetraplegic and 5 tetraparetic; ventilation was not required in any cases. Urinary dysfunction was seen in 12 cases and sensory disturbance in 20 patients.

Single-pulse TMS is typically utilized to study corticospinal tract characteristics. ${ }^{6}$ Thus, in our study, we used single-pulse protocol. TMS was performed according to the currently accepted standard procedures. ${ }^{8}$ Patients were seated comfortably in a chair in a well-lit room. We used circular coil $90 \mathrm{~mm}$ in diameter and Neiro-MS-D monophasic current pulse magnetic stimulator (Neurosoft, Ivanovo, Russia). Single-pulse TMS protocol was used. Coil was positioned over the optimal scalp site: over the vertex for the hand motor cortex stimulation and $5 \mathrm{~cm}$ in front for the leg motor cortex stimulation. Cortical motor areas, cervical and lumbar parts of the spine were stimulated to obtain motor evoked potentials (MEPs) and to calculate central motor conduction times (CMCTs). The target muscles ( $m$. Abductor hallucis) were at rest to ensure same protocol for plegic and paretic patients. The onset of the MEP was determined by measuring the shortest latency of at least 5 single-stimulation responses. For each site, at least 5 MEPs were collected, averaged and analyzed. MEP amplitude, duration and onset latency were measured. MEP amplitude was calculated by the area under the curve. The

${ }^{1}$ Department of Functional Diagnostics, Scientific Research Institute of Children's Infections, Federal Medical-Biological Agency of Russia, Saint-Petersburg, Russia and ${ }^{2}$ Department of Neuroinfections, Scientific Research Institute of Children's Infections, Saint-Petersburg, Russia

Correspondence: Dr VB Voitenkov, Department of Functional Diagnostics, Scientific Research Institute of Children's Infections, Popova 9, Saint-Petersburg 197022, Russia. E-mail: vlad203@inbox.ru

Received 27 March 2015; revised 21 May 2015; accepted 27 June 2015; published online 4 August 2015 
measurements were performed for the hands and legs. Recording surface skinmounted electromyography electrodes (diameter $8 \mathrm{~mm}$ ) were placed on $m$. Abductor hallucis. Surface electromyography signal was recorded using a NeiroMVP apparatus.

All TMS patients underwent a thorough neurological investigation, blood and CSF etiology tests and magnetic resonance imaging of the spinal cord. Inclusion criteria were the established diagnosis of viral myelitis, specific lesions on magnetic resonance imaging and the age younger than 18 years. Exclusion criteria were negative magnetic resonance imaging and age over 18 years. TMS was performed on 12-24 day after the onset of the first symptoms, average on 16th day (s.d. 5.4 days).

Study was approved by the local ethical committee according to the declaration of Helsinki principles. The purpose of the study was fully explained to the participants, their parents or legal representatives; written informed consent was obtained from all patients' parents or legal representatives.

\section{Obtained values were compared between groups.}

Statistical analysis was performed by using statistical analysis software for Windows. For the demographic features of the cohort, descriptive statistics were used. For group comparisons, Student's $t$-test was used for normally distributed parameters. A $P$-value of $<0.05$ was considered statistically significant. Statistical analysis and data processing were carried out using the software IBM SPSS Statistics, version 22.0 (IBM Corp., Armonk, NY, USA). ROC analysis (receiver operator characteristic) was used in prognosis of longterm (3-5 years) recovery of the ability to walk.

\section{RESULTS}

TMS parameters (cortical MEP latencies, amplitudes, CMCT) of the patients and the control group are presented in Table 1. Variability of the MEP latencies and CMCT in controls was small.

Three neurophysiologic patterns were observed in the patients with myelitis:

1. Presence of both cortical MEP (although disperse and low amplitude) and spinal MEP. This pattern was seen in 15 patients.

2. Presence of only spinal MEP without cortical one (seen on Figure 1). This was registered in six patients.

3. Total absence of both spinal and cortical MEP. This was observed in three patients.

In the follow-up period, patients were periodically (every 6 months) investigated and underwent neurologic assessment. Follow-up period's

Table 1 TMS parameters in patients with acute transverse myelitis and controls

\begin{tabular}{|c|c|c|c|c|}
\hline \multirow[t]{2}{*}{ TMS values } & \multicolumn{2}{|c|}{$\begin{array}{l}\text { Myelitis patients } \\
\qquad(\mathrm{n}=24)\end{array}$} & \multicolumn{2}{|c|}{ Controls $(n=20)$} \\
\hline & Average & $S D N$ & Average & $S D N$ \\
\hline \multicolumn{5}{|c|}{ MEP latency (ms) } \\
\hline Right leg & 46.9 & 8.35 & 36.73 & 2.39 \\
\hline Left leg & 48.2 & 7.24 & 36.86 & 2.36 \\
\hline \multicolumn{5}{|c|}{ MEP amplitude $(\mu V)$} \\
\hline Right leg & $0.34^{*}$ & 0.48 & 1.52 & 1.14 \\
\hline Left leg & $0.29^{*}$ & 0.51 & 1.32 & 1.09 \\
\hline \multicolumn{5}{|l|}{ CMCT (ms) } \\
\hline Right leg & $27.0^{*}$ & 3.57 & 16.37 & 1.27 \\
\hline Left leg & $28.5^{*}$ & 4.76 & 16.23 & 1.51 \\
\hline
\end{tabular}

Abbreviations: CMCT, central motor conduction time; SDN, Standard deviation; TMS, transcranial magnetic stimulation; MEP, motor evoked potential.

$* P<0.05$ duration was $2-5$ years, average 3.1 years. Ten children (42\%) were unable to walk $50 \mathrm{~m}$ and required a walker or other support, $11(46 \%)$ had normal motor function and $3(13 \%)$ had total paraplegia without any movement of the lower limbs and no signs of recovery.

Although it has to be noted that, among those 11 patients who fully recovered, only the first TMS pattern (presence of both cortical and spinal MEPs) was registered. Those who recovered with sequelae had first or second (only spinal MEP without cortical) pattern present. In three patients who did not recover over the years and had total absence of movements, only the absence of all MEPs was seen.

Additional electromyography performed in these three patients on first stage of the disease showed motor potentials with normal amplitude and conduction velocity. On later stages of the disease in these three patients in all cases peripheral motor potentials vanished without the possibility to induce them both electrically and magnetically.

Also, among those last three patients, lowering of the MEP threshold for the upper extremities was seen; even relatively low coil induction (35-40\%) induced jerky hand movements without any movement of the legs.

On an individual level, in 23 patients (96\%), MEP changes were registered: amplitude lowering was seen in 20 children $(83 \%)$ and latency asymmetry in 16 (67\%); cortical MEP shape was dispersed in all 15 patients, in whom they were seen.

ROC-analysis data revealed that such neurophysiological parameters as MEP amplitude and MEP latency obtained in an acute period of myelitis had no prognostic significance of long-term (3-5 years) recovery of walk. MEP amplitude and MEP latency according to the ROC analysis have a poor predictive power (AUROC <0.7).

Significant correlation between the long-term (3-5 years) recovery of walk in patients with consequences of myelitis and CMCT value $\geqslant 28.7$ (ms) was found. This model showed a good predictive ability (Figure 2, Table 2).

\section{DISCUSSION}

Latency and amplitude changes in our group were significantly different from those in the controls. This corresponds well with the findings on adult population, as it was shown that decreased amplitudes or the absence of MEP and, more often, latency increase were found in inflammatory lesions. ${ }^{7}$

In all cases in which cortical MEP was elicited, it was polyphasic. Such MEP may represent the demielination process in corticospinal tract. ${ }^{9}$ MEP may be polyphasic in early childhood, but later in life it usually acquires adult characteristics. This finding, thus, may reflect altered conduction along the motor pathways due to their inflammation. Cortical MEPs of abnormally low amplitude usually reflect irreversible axonal damage with predominant lesion of slow

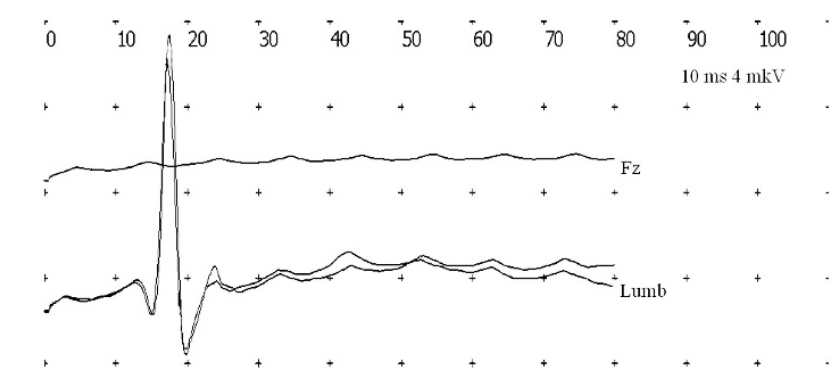

Figure 1 Absence of cortical MEP with normal lumbar MEP in female patient (15-year old) with acute transverse myelitis. 


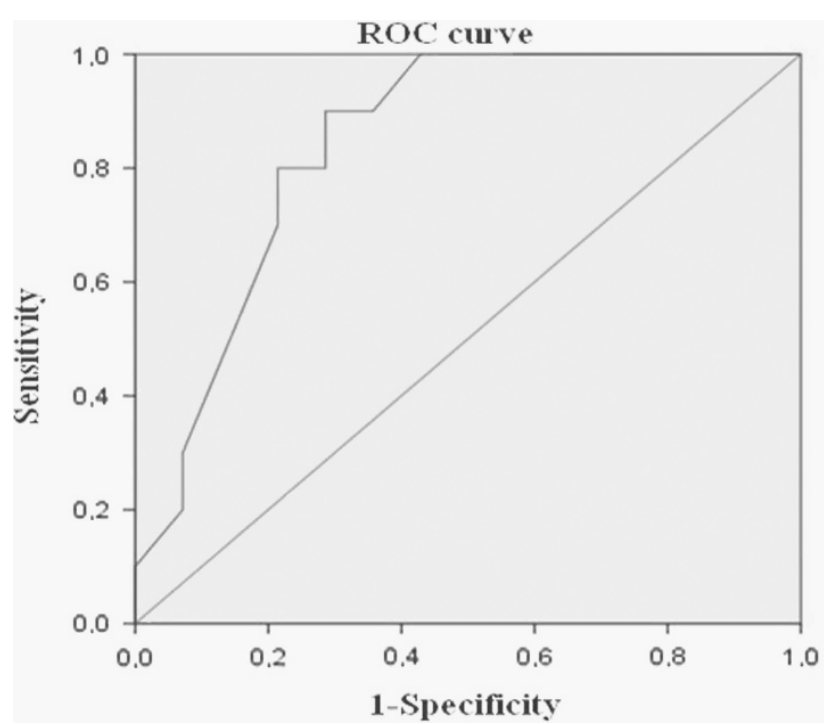

Figure 2 ROC curves derived from the analysis values of CMCT predictive ability of long-term (3-5 years) recovery of walk in patients with myelitis sequelae.

Table 2 Results of ROC analysis of the values of CMCT and MEP amplitude in prognosis of long-term (3-5 years) recovery of walk in patients with myelitis sequelae

\begin{tabular}{lccccc}
\hline Parameters & AUROC & P-value & $\begin{array}{c}\text { Significant } \\
\text { threshold } \\
\text { parameters }\end{array}$ & $\begin{array}{c}\text { Sensitivity } \\
\text { (\%) }\end{array}$ & $\begin{array}{c}\text { Specificity } \\
\text { (\%) }\end{array}$ \\
\hline CMCT & $\mathbf{0 . 8 4 3}$ & $<0.001$ & $\geqslant 28.7$ & 71.4 & 80.0 \\
MEP & 0.071 & 0.8 & $\leqslant 0$ & 58.7 & 75.1 \\
amplitude & & & & & \\
\hline
\end{tabular}

Abbreviations: CMCT, central motor conduction time; MEP, motor evoked potential; ROC, receiver operator characteristic.

Significance for bold values are $P<0.05$.

conductive fibers. ${ }^{9}$ In our patients, more probable explanation may be the temporal lowering of functional state of neurons, as later in the follow-up period MEP amplitudes were elevated in all patients, who had signs of recovery. Cortical MEPs elicited in children with good clinical outcome in the follow-up period correlate well with findings in adults with other kinds of spinal cord injury. When motor responses to TMS are seen after it, this finding is considered to be suggestive of 'root sparing' as a basis for motor function. ${ }^{3,4}$ As may be suggested from the correlation between MEP absence in our group of patients who did not show clinical recovery in the follow-up period and vice versa in those who recovered sufficiently, TMS in this population can identify residual pathways not apparent from clinical assessment alone. This may have a prescriptive value for rehabilitation.

Among other TMS values, which may be of use as prognostic tools in further studies of children with myelitis, are the threshold of MEPs in paravertebral muscles in response to TMS of the motor cortex and the facilitation pattern and latency of MEPs in intercostal muscles during voluntary expiratory effort. ${ }^{10}$ These parameters were not included in our protocol, but this design may significantly improve it in the future works.

\section{CONCLUSIONS}

Acute transverse myelitis in children in $96 \%$ of the cases causes neurophysiologic changes, which may be detected by diagnostic TMS. In this population the method may be used as a predicting tool: absence of the cortical and spinal MEP may be considered as a sign of highly probable poor clinical outcome.

\section{DATA ARCHIVING}

There were no data to deposit.

\section{CONFLICT OF INTEREST}

The authors declare no conflict of interest.

1 Irani ND. Aseptic meningitis and viral myelitis. Neurol Clin 2008; 26: 635-654.

2 Deiva K, Absoud M, Hemingway C, Hernandez Y, Hussson B, Maurey H et al. Acute idiopathic transverse myelitis in children: early predictors of relapse and disability. Neurology 2015; 84: 341-349.

3 Nardone R, Höller Y, Thomschewski A, Höller P, Bergmann J, Golaszewski S et al. Central motor conduction studies in patients with spinal cord disorders: a review. Spinal Cord 2014; 52: 420-427.

4 Nardone R, Hollera Y, Brigob F, Oriolib A, Tezzonb F, Schwenkera K. Descending motor pathways and cortical physiology after spinal cord injury assessed by transcranial magnetic stimulation: a systematic review. Brain Res. (e-pub ahead of print).

5 Barker AT, Jalinous R, Freeston IL. Non-invasive magnetic stimulation of human motor cortex. Lancet 1985; 11: 1106-1107.

6 Frye RE, Rotenberg A, Ousley M, Pascual-Leone A. Transcranial magnetic stimulation in child neurology: current and future directions. J Child Neurol 2008; 23: 9-96.

7 Linden D, Berlit P. Magnetic motor evoked potentials (MEP) in diseases of the spinal cord. Acta Neurol Scand 1994; 90: 348-353.

8 Rossini PM. Clinical application of magnetic transcranial stimulation in multiple sclerosis. In: Lissens MA (ed). Clinical Applications of Magnetic Transcranial Stimulation. Peeters Press: Leuven, Belgium, 1992, pp 21-31.

9 Rajapakse T, Kirton A. Non-invasive brain stimulation in children: applications and future directions. Trans/ Neurosci 2013; 4: 128-137.

10 Ellaway PH, Catley M, Davey NJ, Kuppuswamy A, Strutton P, Frankel HL et al. Review of physiological motor outcome measures in spinal cord injury using transcranial magnetic stimulation and spinal reflexes. J Rehabil Res Dev 2007; 44: 69-76. 